\title{
Ostomy Telehealth for Cancer Survivors: Design of the Ostomy Self-Management Training (OSMT) Randomized Trial
}

\author{
Virginia Sun, PhD, RN ${ }^{1}$, Elizabeth Ercolano, DNSc, MSN², Ruth McCorkle, PhD, RN, FAAN ${ }^{2}$, \\ Marcia Grant, PhD, RN, FAAN ${ }^{1}$, Christopher S. Wendel, $\mathrm{MS}^{3}$, Nancy J. Tallman, RN, BSN, \\ WOC Nurse ${ }^{4}$, Frank Passero, $\mathrm{MD}^{5}$, Sabreen Raza, CCRC ${ }^{6}$, Zuleyha Cidav, $\mathrm{PhD}^{7}$, \\ Michael Holcomb, $\mathrm{BS}^{8}$, Ronald S. Weinstein, $\mathrm{MD}^{8}$, Mark C. Hornbrook, $\mathrm{PhD}^{9}$, \\ Robert S. Krouse, MD, FACS ${ }^{10}$
}

\author{
Author Affiliations: \\ ${ }^{1}$ Division of Nursing Research and Education, Department of Population Sciences, City of Hope, \\ Duarte, CA \\ ${ }^{2}$ School of Nursing, Yale University, New Haven, CT \\ ${ }^{3}$ University of Arizona, Tucson, AZ \\ ${ }^{4}$ Unaffilated, Wound, Ostomy, and Continence Nurse, Tucson, AZ \\ ${ }^{5}$ Unaffiliated, Patient Stakeholder, Philadelphia, PA \\ ${ }^{6}$ Department of Surgery, University of Pennsylvania, Philadelphia, PA \\ ${ }^{7}$ University of Pennsylvania, Philadelphia, PA \\ ${ }^{8}$ Arizona Telemedicine Program, University of Arizona, Tucson, AZ \\ ${ }^{9}$ Kaiser Permanente Center for Health Research, Portland, OR \\ ${ }^{10}$ Corporal Michael J. Crescenz Veterans Affairs Medical Center and Perelman School of \\ Medicine, University of Pennsylvania, Philadelphia, PA
}

Correspondence: Virginia Sun, PhD, RN, Assistant Professor, Division of Nursing Research and Education, Department of Population Sciences, City of Hope, 1500 East Duarte Road, Duarte, CA 91010, USA; Tel: 626/218-3122; fax: 626/218-8941; email: vsun@coh.org 


\begin{abstract}
Purpose: An ostomy adversely affects health-related quality of life (HRQOL) in a diverse population of cancer survivors and their caregivers. Hit-or-miss ostomy care, nurse counseling, and community referral have been the primary modes of self-management education and support in the peri-operative setting. Few evidence-based, systematic ostomy self-management programs are available to ensure optimal post-operative care. This paper describes the study design of a telehealth-based Ostomy Self-Management Training (OSMT) program for cancer survivors and their caregivers.
\end{abstract}

Methods: The study is a three-year, randomized trial that tests the effectiveness of the OSMT program on survivor activation, self-efficacy, and HRQOL. The intervention integrates goal setting and problem-solving approaches to enhance survivor activation and self-efficacy to carry out ostomy care. The curriculum is delivered via four group sessions administered by trained ostomy certified nurses (WOCNs) and peer ostomates. An additional session is offered to caregivers to address their needs in relation to ostomy care. Telehealth approaches through videoconferencing are used to enhance program delivery to participants in three different geographic areas across two time zones. Participants join sessions via real-time videoconferencing from their homes.

Conclusions: The OSMT program has high potential to make a positive impact on the unique physical, psychological, social, and spiritual needs of cancer survivors living with a permanent ostomy. The study design, process, and telehealth approach contributes to the success of future dissemination efforts of the intervention into diverse clinical and community settings. 
Key Words: Cancer; ostomy; quality of life; telehealth; self-efficacy; survivorship

\section{Introduction}

There are over one million individuals in the U.S. living with an ostomy [1], and over 130,000 ostomy surgeries occur annually to treat rectal, bladder, gynecologic, and other gastrourinary cancers [2]. An ostomy refers to the surgically created opening in the body for the discharge of body wastes. For cancer, ostomies are most commonly placed for rectal cancers (colostomy, ileostomy), followed by bladder cancers (urostomy). Ostomies may be needed in other cancers for the management of bulky, metastatic disease or for emergencies such as perforation or obstructions. In $90 \%$ of low-mid rectal cancers, an anastomosis is accompanied by a temporary ("protective”) diverting ileostomy [3]; this is later reversed in a second surgery. A temporary ostomy allows the anastomosis to heal, and prevents catastrophic complications. In some cases, temporary ostomies may become permanent long-term as a result of underlying comorbidities and complications, such as anastomotic leaks or strictures. The treatment decisionmaking process for ostomy surgery is complex. It involves treatment intent, careful consideration of the functional sequelae of surgery, and whether to maintain or restore bowel or urinary continence.

Multiple previous studies, including those conducted by our research team, have shown that an ostomy poses significant health-related quality of life (HRQOL) issues for survivors. After surgery, survivors must adjust to major changes with their anatomy, including life-long modifications to their daily body functions [4]. Our previous population-based observational research with survivors from Kaiser Permanente (KP) and Veterans Affairs (VA) systems suggests that ostomy impacts all aspects of HRQOL, including physical, psychological, social, and spiritual well-being [5-7]. Physically, survivors experience persistent issues with ostomy 
self-care [8-10] and late and long-term complications [11-13]. Psychologically, depressive symptoms are prevalent in ostomy survivors, and we have reported a prevalence rate of $31 \%$ in ostomy survivors [14]. Socially, intimacy and sexual function issues are common [15-17], and survivors often isolate themselves in an attempt to avoid embarrassing accidents and situations in public $[8,18]$. Ostomies often interfere with work/employment [19] as well as recreation and sporting activities [20]. Spiritually, resilience is a common theme that influences adjustments and adaptations to living with an ostomy [21, 22]. Finally, the impact of an ostomy on caregivers' well-being is also significant, as many caregivers assist survivors with ostomy care [23, 24].

In the absence of evidence-based care to support survivors and families, ostomy care can vary tremendously. Educational content is often neither systematic, nor grounded in self-efficacy and skills building. In addition, multiple factors serve as barriers for access to support programs and resources; these include distance to travel, financial restrictions, lack of transportation, lack of consistent follow-up, lack of surgeon focus on ostomy-related problems, and lack of access to a certified ostomy nurse (WOCN). Given these challenges, it is imperative to design and test interventions for ostomy cancer survivors and their families to improve survivors' well-being. The purpose of this paper is to describe the design of a telehealth-based intervention to support ostomy self-management skills building for cancer survivors and their caregivers.

\section{Materials and Methods}

\section{Study Design}

The Ostomy Telehealth for Cancer Survivors study is a 3-year, multi-site, randomized controlled trial of 162 cancer survivors with ostomies and their caregivers. The trial is designed to test a telehealth-based group intervention of an ostomy self-management training (OSMT) program that aims to promote self-efficacy, adjustment and coping with an ostomy, comparing 
the OSMT group with a usual care (UC) group. The primary aim is to determine whether activation, self-efficacy, ostomy-related knowledge, and HRQOL will be improved and sustained over time. The secondary aim is to determine the differences in ostomy-related medical care utilization and family financial burden imposed on survivors and families.

\section{Study Population}

\section{Eligibility Criteria}

The study aims to enroll cancer survivors over 21 years of age who are at least six weeks after a surgical procedure that necessitated the creation of an intestinal stoma (fecal or urinary). Participants are eligible to participate in the study at least 6 weeks after a surgical procedure that needed an intestinal stoma (fecal or urinary); interventions are initiated at least 6 weeks from surgery. Survivors with temporary ostomies are included, and there is no maximum time since surgery. Caregivers over 21 years of age are also eligible for the study. We define caregivers as a family member (i.e. spouse, daughter/son) or friend identified by the survivor as the individual providing the majority of care. For survivors, having an identified caregiver is not a requirement for eligibility.

\section{Recruitment Strategies}

Participants are enrolled at three study sites: University of Pennsylvania (Philadelphia, PA), Yale University (New Haven, CT), and City of Hope (Duarte, CA). Each of the participating sites has a large catchment area which includes rural and inner-city populations. Participants are recruited at each site through several mechanisms. These include identification of potential participants via 1) institutional registry, records, and databases; 2) referrals from site certified wound, ostomy, and continence nurses (WOCNs); 3) referrals from attending surgeons 
and other clinical providers; and 4) through distribution of Institutional Review Board (IRB) approved study brochures in the community.

\section{Randomization}

Participants who meet eligibility criteria are invited to enroll in the randomized trial. Informed consents for both survivors and caregivers are signed and received prior to randomization. Once consented, the participants are randomized to OSMT or UC, using computer-generated random lists blocked by site and gender. Randomization results are kept in sealed envelopes blinded to investigators and coordinators until assignment. Each participating site has a unique pair of computer-generated randomization lists, one for male and the other for female, ensuring balance by sex between groups. Randomization schema is comprised of blocks of four with permuted patterns so that balance is achieved. Survivors randomized to the OSMT group designates a caregiver (if one is available) upon registration. Baseline assessment surveys are completed following informed consent and randomization. For caregivers, basic sociodemographic information are obtained, including age, gender, relationship to survivor, and current work status.

\section{Study Intervention}

\section{Development of the Intervention}

The development of the OSMT program is described elsewhere [25]. Briefly, the OSMT program is based on the Chronic Care Model (CCM). The CCM transforms what is currently a reactive health care system into one that keeps patients as healthy as possible through planning, proven strategies, management, and patient activation [26, 27]. CCM interventions result in healthier patients, more satisfied providers, and reduced utilization of system resources and medical costs. The OSMT incorporates four essential elements of the CCM that promotes high- 
quality chronic disease management; these include 1) identification and compilation of culturally appropriate resource compendium for ostomy survivors and families; 2) promotion of survivor well-being through planning, proven strategies, and culturally appropriate care; 3) provision of self-efficacy enhancing, evidence-based self-management information and peer support; and 4) utilization of volunteer peer educators for intervention delivery in a telehealth, support group approach.

The self-management component of the OSMT program is based upon the Chronic Disease Self-Management Program established by Lorig and colleagues [28-30]. It incorporates patient activation, defined as taking action to maintain and improve one's health, staying the course even when under stress, and not giving up in the face of setbacks or challenges [31, 32]. Whereas traditional patient education offers information and technical skills, self-management coaching enhances self-efficacy, teaches problem-solving and goal setting skills, and promotes cognitive restructuring. A second central concept in self-management is self-efficacy, which is defined as confidence to carry out a behavior necessary to reach a desired goal [33]. OSMT program sessions are highly participatory, and promote mutual support for building participants' confidence in their ability to manage their ostomies.

A longitudinal, single-site, single-group design pilot study (NCI R21 CA133337) was conducted to test the feasibility of the OSMT program $(\mathrm{N}=38)$. The majority of participants had a history of rectal cancer (60.5\%) or bladder cancer (28.9\%) [34]. Mean age was 71.3 (Range 6082), and participants were predominantly male (73.7\%). The majority of participants were white (non-Hispanic); the most common diagnosis was colorectal (60.5\%), followed by bladder/prostate (28.9\%), and ovarian (2.6\%). More than half of participants had a 
colostomy/ileostomy (52.7), and 28.9\% had a urostomy. The average time since surgery was 201 days (range 22 to 1,626). A total of 22 family caregivers received the intervention.

The primary endpoint for the pilot study was to examine change in outcome scores from pre- to post-intervention through paired $t$-tests. Overall, outcome measures improved pre- and post-intervention, and they appeared to be sustained (Table 2). Scores were significantly improved and sustained for patient activation ( $\mathrm{p}=0.001$ ) and self-efficacy for ostomy care ( $\mathrm{p}=0.008)$ [34]. Total HRQOL ( $\mathrm{p}=0.03)$, physical well-being $(\mathrm{p}=0.01)$, and social well-being $(p=0.005)$ were also significantly improved. The significant improvement in patient activation was clinically meaningful (an average of nearly 11 point improvement); a 3-point change in standard errors is considered clinically important [35]. The majority of participants had a very positive experience with the OSMT program. A major challenge of the pilot study, as reported by participants, was difficulty in traveling to attend the group sessions. The telehealth design for the randomized trial was selected to overcome this challenge by allowing participation without traveling to a centralized location.

\section{Intervention Content and Delivery}

The OSMT program is divided into five sessions: three for survivors, one for caregivers, and the last as a booster session for both survivor and caregiver participants (Table 1). Each session takes approximately two hours to complete. Participants receive a program workbook to guide session participation, as well as a site-specific resource list that contains both institutional

and community resources. The resource list is reviewed and discussed at the end of each session. Prior to Session 1 for survivors, participants list personal goals for participation in the program. These goals are reviewed and assessed for progress at subsequent sessions. The focus of Session 1 is on understanding ostomy self-care, equipment, appliances, and stoma/skin care. Assignment 
for the session is to monitor ostomy output (frequency and consistency of bowel movements and flatus), and problems related to pouching system. Teaching methods are interactive using mixed media presentations (i.e. slide presentations), and hands on practice with equipment, pouches, and belts. In Session 2 for survivors, the focus is on social well-being concerns, including social/interpersonal relationships, intimacy, sexuality, and communicating with family and friends. Management of co-morbidities and other long-term effects of treatment (i.e. fatigue) are discussed. Problem-solving skills training focuses on creating an emergency kit for public outings. Caregivers attend a separate session that covers the same topics in Sessions 1 and 2 for survivors. In addition, the caregiver session also include support for caregiver adjustment and improving comfort level with ostomy care. In Session 3 for survivors, the program promotes a healthy lifestyle through nutritional management, physical activity recommendations, psychological health, and improving attitudes. Problem-solving training focus on tips for ostomy care while traveling. The final session is a booster session for both survivors and caregivers, where discussions are driven by group demands and remaining questions. Attainment of personal goals for participation in OSMT program is discussed.

In accordance with the principles of self-management and findings from our previous observational studies, the OSMT program is administered by a trained WOCN and peer ostomate. The program's lead WOCNs (N.T. and C.S.) and Peer Ostomate (R.I.) administer each telehealth session from the University of Arizona site located in Tucson, Arizona. In addition, WOCNs at each participating site attend each session to serve as added resources for participants and assist with facilitating the group sessions. Two gender-specific Peer-Ostomates are employed at each site to assist with group sessions, as well as serve as assigned "buddies" for participants. They are paired with survivors by gender; this is to promote discussion of sensitive 
issues where it might be uncomfortable to talk about with someone of the opposite sex. In our pilot study experience, participants rarely contacted the Peer-Ostomates, but their opinion was actively sought during the sessions.

\section{Telehealth Delivery/Procedures}

Telehealth is the access point to health care for millions of Americans.

Videoconferencing, transmission of still images, e-health including patient portals, remote monitoring of vital signs, web-based continuing medical education, and nursing call centers are all considered part of telehealth [36]. Technology design and support for this telehealth intervention is provided by the Arizona Telemedicine Program (ATP), based at the University of Arizona in Tucson, Arizona. Upon informed consent, participants randomized to the OSMT group consult via telephone with ATP staff to review the technical requirements for participation in the telehealth sessions. Participants must have access to a computer, laptop, tablet or smartphone equipped with a webcam, microphone, and speaker that meets or exceeds the requirements specified by the Zoom video conferencing service. Zoom was selected as the video conferencing platform for many reasons including HIPAA compliant security, quality and stability of video and audio connection, ease of use and its cross-platform support for leading mobile device and computer operating systems. Participants that do not have access to the required technology or internet connectivity are offered a temporary loan of tablets and mobile hotspots. A signed loan agreement is requested prior to borrowing a tablet. The tablet and/or mobile hotspot, required accessories, and a pre-paid return shipping label are shipped to the participant. All participants complete a test video call with ATP helpdesk staff via Zoom to assess their ability to successfully communicate via Zoom video conferencing. The helpdesk staff also work with participants to solve any network connection, hardware or software 
problems, and orient participants to Zoom software features such as microphone and camera controls, and gallery and speaker views. Assuring each participant's ability to connect successfully, and familiarizing each participant with Zoom software features necessary for connection, are essential preparations to ultimately engaging participants in a seamless interactive education experience during each live OSMT education video conferencing session. These preparations allow each OSMT cohort to maximally focus on learning and peer support with minimal distraction due to technical problems. All OSMT group session participants connect via video conferencing and are visible on camera to the rest of the group. Phone (nonvideo) participation is not allowed. Access to a private and relatively quiet environment is encouraged during the telehealth sessions.

A new telehealth cohort begins each month. Based on our pilot study experience, each session includes a maximum of six study participants, in addition to Peer Ostomates and WOCNs, for optimal participation in a group setting. Participants receive reminder calls one day prior to each of the training sessions. The telehealth sessions are not recorded for privacy purposes. All video conferencing sessions use a HIPAA compliant Zoom account acquired and managed by the ATP. An ATP staff member attends each video conferencing session in person at the same location as the WOCNs to provide technical support for all throughout the session.

In addition to providing interventionists and participants the ability to see and hear each other in user selectable gallery or full screen views, Zoom also provides the ability for WOCNs to share content such as educational diagrams, photos and information. The pan-tilt-zoom video camera used by the lead WOCNs in Tucson enables the WOCNs to share close-up views of ostomy appliances, accessories and resources. ATP staff supporting each group session use Zoom's video conferencing management controls to assist interventionists and participants with 
Zoom features as needed throughout each call. It is notable that study investigators, educators and support personnel hold their weekly meetings via Zoom video conferencing. This practice provides study personnel with ongoing experience utilizing the Zoom meeting platform, which results in distributed expertise on commonly used Zoom features, and enables them to assist new participants and peers directly and immediately with many routine questions about Zoom.

\section{Usual Care Condition}

Participants randomized to the UC group receive the following: 1) recommendation for at least one encounter with a WOCN; 2) recommendation for at least one post-operative follow-up visit with the surgeon; 3) a booklet published by The Wound, Ostomy, and Continence Nurses' Society entitled "Basic Ostomy Skin Care: A Guide for Patients and Health Care Providers”; 4) local and national ostomy support group contact information; 5) contact information for site nutritional and physical therapists; and 6) site WOCN contact information. If participants have specific questions about the materials, site research coordinators clarify the content and encourage the participants to call their physician or WOCN about any continuing problem. These resources are also provided to those randomized to the OSMT program.

\section{Outcome Measures and Data Management}

Surveys were chosen to be patient-centered, validated, and self-management theorydriven. The estimated time to complete the surveys is about 50 minutes. All survivors receive a remuneration of $\$ 150$ for study participation, to be distributed at the completion of each of the three data collection time points. For caregivers in the OSMT group, a remuneration of \$25 is provided after completion of the two caregiver telehealth sessions (total of \$50). Data are collected for all participants at baseline (pre-intervention), the end of session 4 (immediate postintervention), and at month 7, (six months post-intervention) using the following measures: 
Patient Activation Measure (PAM): The PAM uses a uni-dimensional, probabilistic Guttmanlike scale that reflects a developmental model of activation that involves four stages: 1) believing the patient role is important, 2) having the confidence and knowledge necessary to take action, 3) actually taking action to maintain and improve one's health, and 4) staying the course even under stress. The measure can be used at the individual patient level to tailor interventions and assess changes. Those with higher activation scores report significantly better health, and significantly lower rates of doctor office visits, emergency room visits, and hospital nights. High internal consistency (alpha .87) and construct validity have been reported [31, 32, 37]. Self-Efficacy to Perform Ostomy Self-Management Behaviors: Modified from Lorig and colleagues’ outcome measure for Chronic Disease Management Interventions, this survey represents 8 domains (with reported Cronbach's alpha for original scales: physical activity (.83), information seeking (NA), support (.77), communication with HC providers (.90), ostomy management (.87), social and recreational (.82), symptom management (.91), and depression (.92) [28-30]. The tool contains a total of 35 items, with items scaled from 1-10, with higher scores representing higher self-efficacy across the 8 domains.

City of Hope-Quality of Life-Colorectal (COH-QOL-CRC): This survey was designed for all adult ostomates, and has a 4-dimensional HRQOL (physical, psychological, social and spiritual well-being) framework assessing demographic, personal (e.g. diet, work, and activity) and clinical data. It has an open-ended question of the greatest challenge for ostomy survivors. A multi-item scale section assesses overall HRQOL and the four HRQOL dimensions on a 0 to 10 scale. The full survey is used at baseline; for post intervention and follow-up, only the 43 scaled items (those items sensitive to change over time) is used. Reported Cronbach's alphas are .94 total scale and $.80-.90$ subscales [7, 38, 39]. 
Ostomy Knowledge Questionnaire: This 11-item survey contains multiple choice questions to assess pre- and post-intervention knowledge related to OSMT program content and ostomy care [39].

Hospital Anxiety and Depression Scale (HADS): The HADS is a self-assessment instrument for detecting anxiety and depression in medical outpatients, which has demonstrated sensitive in community and in-patient cancer studies, with subscales of $<7$ not present, 8-10 doubtful, $>11$ definite. Reported Cronbach’s alpha .84-.89 [40].

Burden of Ostomy Care Tool: Based on the model developed by Given and colleagues for longterm care [41], this survey gathers participant self-reports of ostomy-related medical care use, productivity losses, and associated out-of-pocket expenses and time spent for ostomy care in general, and the OSMT intervention, caregivers, and paid housekeeping. The tool contains a total of 35 items that assesses the following endpoints: ostomy-specific family resource use, ostomyspecific health care resource use, ostomy-specific family financial burden, types and level of effort for intervention personnel, and specific equipment, supplies, and informatics support required to implement the study.

Project coordinators at each site are responsible for data collection, auditing, and data entry. Surveys are available to complete in both paper format or electronically via the web using REDCap ${ }^{\circledR}$ (Research Electronic Data Capture). Site coordinators provide their respective subjects with their unique survey ID and survey web link at the appropriate time. When completed electronically, the survey responses transmits directly to the RedCap ${ }^{\circledR}$ server at the University of Arizona.

\section{Quality Assurance (QA) and Monitoring}


QA efforts include the development and use of interventionist training manuals. This process ensures intervention fidelity for all telehealth sessions. Regular weekly meetings are held via Zoom videoconferencing and include the PI, site PIs, co-investigators, consultants, WOCNs, Peer Ostomates, and Project Coordinators. The meetings are held to update overall accrual and other key trial issues (i.e. eligibility criteria, data collection, data entry).

In accordance with funding agency requirements, a Stakeholder Advisory Council (SAC) is established for the randomized trial. The SAC is designed to ensure integrity and focus of the study. It also serves as a resource for accrual issues, unforeseen problems, and interpretation of results. A well-known role of Patient Advocacy groups in cooperative groups is to troubleshoot accrual issues and assist in finding practical solutions. In addition, unforeseen participant concerns are bound to appear, and an expert panel that meets regularly, as well as on an emergency basis, is an advantageous resource to have in a multi-site study.

The SAC is composed of ostomy nurses, peer ostomates, patients, and caregivers who have participated in the OSMT program. The PI, site PIs, co-investigators, and consultants do not participate in the SAC. There is a maximum of six members of the SAC, with an equitable distribution of patients, caregivers, and WOCNs. The group determines its chairperson, who is expected to report to the PI and Research Team. A telephone conference line is established for the quarterly meetings of the SAC over the 3-year project. The SAC minutes are circulated to the Research Team, and the SAC leader presents them at the next Research Team meeting. Feedback to the Research Team occurs via minutes from the SAC.

\section{Discussion}

For cancer survivors, living with an ostomy significantly impacts all aspects of HRQOL. To our knowledge, no randomized trial in cancer survivorship has yet been conducted to promote 
survivor and caregiver self-management and adaptations to living with an ostomy. Interventions to build self-efficacy and attend to the physical, psychosocial, and spiritual consequences of surgery are imperative in order to achieve the best possible outcomes for ostomy survivors and their families [38, 42]. Our project is timely, innovative, and unique for several reasons. First, more than 15.5 million Americans are living with a history of cancer; this number is projected to grow to 20.3 million by 2026 [43]. Many challenges remain for cancer survivors, despite increasing awareness of survivorship issues after treatment. A major challenge is the lack of evidence-based guidelines and interventions for quality post-treatment care. Ongoing efforts to identify interventions for cancer survivors are needed to address these challenges. The OSMT program addresses the unique HRQOL challenges for cancer survivors living with an ostomy, and can serve as a national model for comprehensive, quality survivorship care.

Second, the OSMT program has great potential for improving HRQOL and outcomes for cancer survivors because it is grounded in the CCM and self-management models, two evidencebased theoretical frameworks. The CCM can be applied to a variety of chronic illnesses (including cancer survivorship), health care settings, and target populations. Self-management education complements traditional patient education in supporting survivors to live the best possible HRQOL with their ostomy. Ostomy self-management entails appropriate education to ensure survivors and their caregivers have a well-rounded knowledge of issues, understanding of methods to address these issues, and resources available when questions and concerns arise. The intervention was pilot-tested with proven preliminary efficacy, and has been modified by a group of cancer survivorship, chronic disease, ostomy, patient activation and education experts to respond to the specific needs of ostomates from diverse backgrounds, abilities, and resources. 
Third, our telehealth approach in delivery of the intervention is innovative and timely. The OSMT program has been designed to accommodate adult education and distance learning concepts. Electronic communication from home utilizing telehealth technologies, protocols, and techniques offers many advantages, including greater access, affordability, and convenience. Many health-related issues, including those for survivors with ostomies, can easily be managed via electronic communication. Furthermore, telehealth is widely recognized as a useful, important healthcare option for underserved populations. Those with access disparities, such as rural populations, elderly, inner-city, and ill patients, commonly have difficulty accessing specialty care. Telehealth offers the opportunity for healthcare providers to care for patients who are unable to access high-quality face-to-face care in an affordable, convenient manner. Finally, the telehealth approach also provides a unique opportunity for simultaneous delivery of a standardized program curriculum across three different geographic areas and two different time zones. This wide coverage and inclusivity enhances the generalizability of future findings from the randomized trial.

Finally, several components of our trial design were included to prepare for large-scale dissemination and implementation following trial completion. These include: 1) an intervention operations manual; and 2) official versions of all intervention materials for interventionists and participants. Our proposed replication model includes the incremental physical resources required to implement the OSMT program. Interventionist and research staff capture the following data: 1) preparation time incurred for video-conferencing presentations and handouts; 2) session attendance records; 3) marketing and advertising activities for participant recruitment; 4) videoconferencing preparation and set-up time, direct contact time, and post-conferencing wrap-up; 5) peer ostomate mentoring time (number of contacts, duration of each contact, and 
time required to chart each contact); 6) intervention IT and videoconferencing equipment; and 7) staff time spent helping participants with videoconferencing set-up. Our long-term goal is to have the OSMT program entrenched in communities so that it can be readily translatable to diverse clinical settings and community-based programs.

In summary, the OSMT randomized trial aims to test the effectiveness of a selfmanagement-enhancing telehealth intervention to improve outcomes for cancer survivors living with an ostomy and their caregivers. Overall, the study has a high probability of success because it is built on solid theoretical and practical understanding of the issues facing survivors with ostomies and is broad-based, adaptable, accessible, simple, low-intensity, and reproducible.

Acknowledgements: Research reported in this article was funded through a Patient-Centered Outcomes Research Institute (PCORI) Award (1507-31690). The statements presented in this article are solely the responsibility of the author(s) and do not necessarily represent the views of the Patient-Centered Outcomes Research Institute (PCORI), its Board of Governors or Methodology Committee.

The OSMT Trial Group 
University of Pennsylvania: Robert S. Krouse, MD, FACS, Zuleyha Cidav, PhD, Nancy J. Tallman, RN, BSN, WOC Nurse, Christie Simons, BSN, WOCN, Ave Maria Preston, MSN, RN, CWON, ACNS-BC, Matthew Ciabattoni, BSN, RN-BC, CWOCN, Sabreen Raza, CCRC, Roger Iverson, Frank Passero, MD, Deborah Donahue, Marcia Grant, PhD, RN, FAAN, Mark C. Hornbrook, PhD, Judith H. Hibbard, DrPH, MPH, Mary Wagner

University of Arizona: Ronald Weinstein, MD, Christopher S. Wendel, MS, Michael Holcomb, BS, Pete Yonsetto, Phyllis Webster, Octavio Bojorquez, BS

City of Hope: Virginia Sun, PhD, RN, Lily Lai, MD, Anne Marie Whaley, FNP-C, MSN, BA, CWOCN, CFCN, PHN, Nataki Tomt, MA, RN, BSN, CWON, Anne Marie Knudsen, MSN, RN, CWOCN, Lynn MacDougall

Yale University: Ruth McCorkle, PhD, RN, FAAN, Elizabeth Ercolano, RN, MSN, DNSc, Lucinda Pinchot, RN, BSN, WOCN, Johnathan Friedlaender, PhD, Pamela Pitcher, Mary Ellen Clancy, RN, ADN

\section{References}

[1] United Ostomy Association of America, 2017. www.ostomy.org. (Accessed May 27 2017).

[2] R.L. Siegel, K.D. Miller, A. Jemal, Cancer Statistics, 2017, CA: a cancer journal for clinicians 67(1) (2017) 7-30. 
[3] J.W. Fleshman, N. Smallwood, Current concepts in rectal cancer, Clinics in colon and rectal surgery 28(1) (2015) 5-11.

[4] S. Kenderian, E.K. Stephens, A. Jatoi, Ostomies in rectal cancer patients: what is their psychosocial impact?, European journal of cancer care 23(3) (2014) 328-32.

[5] R.S. Krouse, L.J. Herrinton, M. Grant, C.S. Wendel, S.B. Green, M.J. Mohler, C.M. Baldwin, C.K. McMullen, S.M. Rawl, E. Matayoshi, S.J. Coons, M.C. Hornbrook, Health-related quality of life among long-term rectal cancer survivors with an ostomy: manifestations by sex, Journal of clinical oncology : official journal of the American Society of Clinical Oncology 27(28) (2009) 4664-70.

[6] R.S. Krouse, M. Grant, C.S. Wendel, M.J. Mohler, S.M. Rawl, C.M. Baldwin, S.J. Coons, R. McCorkle, C.Y. Ko, C.M. Schmidt, A mixed-methods evaluation of health-related quality of life for male veterans with and without intestinal stomas, Diseases of the colon and rectum 50(12) (2007) 2054-66.

[7] R. Krouse, M. Grant, B. Ferrell, G. Dean, R. Nelson, D. Chu, Quality of life outcomes in 599 cancer and non-cancer patients with colostomies, J Surg Res 138(1) (2007) 79-87.

[8] V. Sun, M. Grant, C.K. McMullen, A. Altschuler, M.J. Mohler, M.C. Hornbrook, L.J. Herrinton, C.M. Baldwin, R.S. Krouse, Surviving colorectal cancer: long-term, persistent ostomy-specific concerns and adaptations, Journal of wound, ostomy, and continence nursing : official publication of The Wound, Ostomy and Continence Nurses Society 40(1) (2013) 61-72.

[9] C.K. McMullen, M.C. Hornbrook, M. Grant, C.M. Baldwin, C.S. Wendel, M.J. Mohler, A. Altschuler, M. Ramirez, R.S. Krouse, The greatest challenges reported by long-term 
colorectal cancer survivors with stomas, The journal of supportive oncology 6(4) (2008) $175-82$.

[10] C.K. McMullen, J.E. Bulkley, A. Altschuler, C.S. Wendel, M. Grant, M.C. Hornbrook, V. Sun, R.S. Krouse, Greatest Challenges of Rectal Cancer Survivors: Results of a Population-Based Survey, Diseases of the colon and rectum 59(11) (2016) 1019-1027.

[11] J. Pittman, S.M. Rawl, C.M. Schmidt, M. Grant, C.Y. Ko, C. Wendel, R.S. Krouse, Demographic and clinical factors related to ostomy complications and quality of life in veterans with an ostomy, J Wound Ostomy Cont 35(5) (2008) 493-503.

[12] C.K. McMullen, J. Wasserman, A. Altschuler, M.L. Grant, M.C. Hornbrook, P. Liljestrand, C. Briggs, R.S. Krouse, Untreated Peristomal Skin Complications Among Long-Term Colorectal Cancer Survivors With Ostomies, Clin J Oncol Nurs 15(6) (2011) 644-650.

[13] L. Liu, L.J. Herrinton, M.C. Hornbrook, C.S. Wendel, M. Grant, R.S. Krouse, Early and late complications among long-term colorectal cancer survivors with ostomy or anastomosis, Diseases of the colon and rectum 53(2) (2010) 200-12.

[14] Y. Chongpison, M.C. Hornbrook, R.B. Harris, L.J. Herrinton, J.K. Gerald, M. Grant, J.E. Bulkley, C.S. Wendel, R.S. Krouse, Self-reported depression and perceived financial burden among long-term rectal cancer survivors, Psycho-oncology 25(11) (2016) 13501356.

[15] M.R. Symms, S.M. Rawl, M. Grant, C.S. Wendel, S.J. Coons, S. Hickey, C.M. Baldwin, R. Krouse, Sexual health and quality of life among male veterans with intestinal ostomies, Clin Nurse Spec 22(1) (2008) 30-40. 
[16] V. Sun, M. Grant, C.S. Wendel, C.K. McMullen, J.E. Bulkley, L.J. Herrinton, M.C. Hornbrook, R.S. Krouse, Sexual Function and Health-Related Quality of Life in LongTerm Rectal Cancer Survivors, The journal of sexual medicine 13(7) (2016) 1071-9.

[17] M. Ramirez, C. McMullen, M. Grant, A. Altschuler, M.C. Hornbrook, R.S. Krouse, Figuring out sex in a reconfigured body: experiences of female colorectal cancer survivors with ostomies, Women \& health 49(8) (2009) 608-24.

[18] K.A. Mitchell, S.M. Rawl, C.M. Schmidt, M. Grant, C.Y. Ko, C.M. Baldwin, C. Wendel, R.S. Krouse, Demographic, clinical, and quality of life variables related to embarrassment in veterans living with an intestinal stoma, Journal of wound, ostomy, and continence nursing : official publication of The Wound, Ostomy and Continence Nurses Society 34(5) (2007) 524-32.

[19] D.J. Horner, C.S. Wendel, R. Skeps, S.M. Rawl, M. Grant, C.M. Schmidt, C.Y. Ko, R.S. Krouse, Positive correlation of employment and psychological well-being for veterans with major abdominal surgery, Am J Surg 200(5) (2010) 585-90.

[20] C. McMullen, L. Liu, J.E. Bulkley, M.C. Hornbrook, C. Wendel, M. Grant, A. Altschuler, L.K. Temple, R.S. Krouse, L. Herrinton, Participation in Activities Associated With Quality of Life for Long-Term Survivors of Rectal Cancer, The Permanente journal 21 (2017).

[21] J. Bulkley, C.K. McMullen, M.C. Hornbrook, M. Grant, A. Altschuler, C.S. Wendel, R.S. Krouse, Spiritual well-being in long-term colorectal cancer survivors with ostomies, Psycho-oncology 22(11) (2013) 2513-21.

[22] C.M. Baldwin, M. Grant, C. Wendel, S. Rawl, C.M. Schmidt, C. Ko, R.S. Krouse, Influence of intestinal stoma on spiritual quality of life of U.S. veterans, Journal of holistic nursing 
: official journal of the American Holistic Nurses' Association 26(3) (2008) 185-94; discussion 195-6; quiz 197-9.

[23] C.K. McMullen, J. Schneider, A. Altschuler, M. Grant, M.C. Hornbrook, P. Liljestrand, R.S. Krouse, Caregivers as healthcare managers: health management activities, needs, and caregiving relationships for colorectal cancer survivors with ostomies, Supportive care in cancer : official journal of the Multinational Association of Supportive Care in Cancer 22(9) (2014) 2401-8.

[24] A.L. Silva, P.S. Monteiro, J.B. Sousa, A.L. Vianna, P.G. Oliveira, Partners of patients having a permanent colostomy should also receive attention from the healthcare team, Colorectal disease : the official journal of the Association of Coloproctology of Great Britain and Ireland 16(12) (2014) O431-4.

[25] M. Grant, R. McCorkle, M.C. Hornbrook, C.S. Wendel, R. Krouse, Development of a chronic care ostomy self-management program, Journal of cancer education : the official journal of the American Association for Cancer Education 28(1) (2013) 70-8.

[26] E.H. Wagner, S.M. Bennett, B.T. Austin, S.M. Greene, J.K. Schaefer, M. Vonkorff, Finding common ground: patient-centeredness and evidence-based chronic illness care, Journal of alternative and complementary medicine 11 Suppl 1 (2005) S7-15.

[27] E.H. Wagner, B.T. Austin, C. Davis, M. Hindmarsh, J. Schaefer, A. Bonomi, Improving chronic illness care: translating evidence into action, Health affairs 20(6) (2001) 64-78.

[28] K.R. Lorig, D.S. Sobel, A.L. Stewart, B.W. Brown, Jr., A. Bandura, P. Ritter, V.M. Gonzalez, D.D. Laurent, H.R. Holman, Evidence suggesting that a chronic disease selfmanagement program can improve health status while reducing hospitalization: a randomized trial, Medical care 37(1) (1999) 5-14. 
[29] K.R. Lorig, P.D. Mazonson, H.R. Holman, Evidence suggesting that health education for self-management in patients with chronic arthritis has sustained health benefits while reducing health care costs, Arthritis and rheumatism 36(4) (1993) 439-46.

[30] K. Lorig, J. Laurin, H.R. Holman, Arthritis self-management: a study of the effectiveness of patient education for the elderly, The Gerontologist 24(5) (1984) 455-7.

[31] J.H. Hibbard, E.R. Mahoney, J. Stockard, M. Tusler, Development and testing of a short form of the patient activation measure, Health services research 40(6 Pt 1) (2005) 191830.

[32] J.H. Hibbard, J. Stockard, E.R. Mahoney, M. Tusler, Development of the Patient Activation Measure (PAM): conceptualizing and measuring activation in patients and consumers, Health services research 39(4 Pt 1) (2004) 1005-26.

[33] A. Bandura, Self-efficacy: toward a unifying theory of behavioral change, Psychological review 84(2) (1977) 191-215.

[34] R.S. Krouse, M. Grant, R. McCorkle, C.S. Wendel, M.D. Cobb, N.J. Tallman, E. Ercolano, V. Sun, J.H. Hibbard, M.C. Hornbrook, A chronic care ostomy self-management program for cancer survivors, Psycho-oncology 25(5) (2016) 574-81.

[35] J.B. Fowles, P. Terry, M. Xi, J. Hibbard, C.T. Bloom, L. Harvey, Measuring selfmanagement of patients' and employees' health: further validation of the Patient Activation Measure (PAM) based on its relation to employee characteristics, Patient education and counseling 77(1) (2009) 116-22.

[36] American Telemedicine Association, Telemedicine Defined. 
[37] R.L. Skolasky, A.F. Green, D. Scharfstein, C. Boult, L. Reider, S.T. Wegener, Psychometric properties of the patient activation measure among multimorbid older adults, Health services research 46(2) (2011) 457-78.

[38] M.J. Mohler, S.J. Coons, M.C. Hornbrook, L.J. Herrinton, C.S. Wendel, M. Grant, R.S. Krouse, The health-related quality of life in long-term colorectal cancer survivors study: objectives, methods and patient sample, Current medical research and opinion 24(7) (2008) 2059-70.

[39] M. Grant, B. Ferrell, G. Dean, G. Uman, D. Chu, R. Krouse, Revision and psychometric testing of the City of Hope Quality of Life-Ostomy Questionnaire, Quality of life research : an international journal of quality of life aspects of treatment, care and rehabilitation 13(8) (2004) 1445-57.

[40] I.M. Cameron, J.R. Crawford, K. Lawton, I.C. Reid, Psychometric comparison of PHQ-9 and HADS for measuring depression severity in primary care, The British journal of general practice : the journal of the Royal College of General Practitioners 58(546) (2008) 32-6.

[41] B.A. Given, C.W. Given, M. Stommel, Family and out-of-pocket costs for women with breast cancer, Cancer practice 2(3) (1994) 187-93.

[42] Institute of Medicine, Delivering High-Quality Cancer Care: Charting a New Course for a System in Crisis, The National Academies, Washington, DC, 2013.

[43] K.D. Miller, R.L. Siegel, C.C. Lin, A.B. Mariotto, J.L. Kramer, J.H. Rowland, K.D. Stein, R. Alteri, A. Jemal, Cancer treatment and survivorship statistics, 2016, CA: a cancer journal for clinicians 66(4) (2016) 271-89. 
